\title{
Noninvasive measurement of pressure fields arising from arbitrarily-shaped ultrasound transducers
}

\author{
Lizhuo Chen ${ }^{12} 3$, Stefan J. Rupitsch ${ }^{1}$, Reinhard Lerch ${ }^{1}$ \\ 1) Chair of Sensor Technology, University Erlangen-Nuremberg, Paul-Gordan-Str. 3/5, 91052, Erlangen \\ 2) Erlangen Graduate School in Advanced Optical Technologies (SAOT) \\ 3) lizhuo.chen@Ise.e-technik.uni-erlangen.de
}

\begin{abstract}
This paper describes an optical method enabling noninvasive measurement of ultrasound pressure fields underwater. The pressure field arising from a cylindrically focused ultrasound transducer is measured by means of the optical method and a hydrophone. Good agreement has been achieved between the results obtained by these two different methods. After that, the technique is applied on the reconstruction of density change induced by the ultrasound in a PMMA cuboid. The result seems quite plausible, but a quantitative verification is still not possible. Because, so far as we know, no other published experiments have succeeded in the measurement of density change in solid induced by ultrasound.
\end{abstract}

Keywords: noninvasive, ultrasound transducer, pressure field, density change, transparent solid

\section{Introduction}

Currently, ultrasound pressure field underwater is routinely measured by means of hydrophones. In spite of their increasing measurement accuracy and measurement range, hydrophones have to be immersed in water during measurements. The sound diffraction induced by the immersion is often unneglectable. Besides, the output of most hydrophones for detecting high-frequency ultrasound depends strongly on the angle of incidence. To overcome these shortages, we utilize a laser Doppler vibrometer combined with a computed tomography method to reconstruct the ultrasound pressure field underwater. In contrast to hydrophones, this optical measurement is non-perturbing and inherent omnidirectional.

Our previous research was restricted by the assumption of totally axisymmetric sound pressure fields [1]. After the extension of the experimental setup, the ultrasound pressure fields arising from arbitrarily shaped transducers can be measured under different perspectives leading to the possibility of full-angle reconstruction. This new ability is proved through the measurement of ultrasound pressure field arising from a cylindrically focused transducer. Furthermore, using this technique we can also measure the density change within a PMMA (Plexiglas 55) cuboid caused by ultrasound. Such a quantitative reconstruction of density change in solid has not been achieved before.

\section{Theoretical analysis}

\subsection{Change of optical path length}

The measurement setup is mainly composed of an ultrasound transducer, a laser Doppler vibrometer (LDV) and an optical reflector (Fig. 1). A narrow laser beam emitted by the LDV propagates through the ultrasound field arising from the transducer and is reflected back to the sensor head by the optical reflector at the bottom of the water basin. Because of the existence of ultrasound, the refractive index of the medium $n(t)$ at position $(x, y, z)$ is influenced and results in the change of optical path length,

$$
\Delta L(t, x, z)=2 \int_{y_{1}}^{y_{2}} \Delta n(x, y, z, t) \mathrm{d} x,
$$


where $y_{1}$ and $y_{2}$ denote the $y$-coordinates of the reflector and the water surface, respectively (Fig. 1). $\Delta n(x, y, z, t)$ indicates the time-dependent deviation of the refractive index from its original value induced by the ultrasound. The return trip of the laser light has been simplified here with the factor 2 , since the ultrasound field keeps almost constant during the whole time the light travels back and forth.

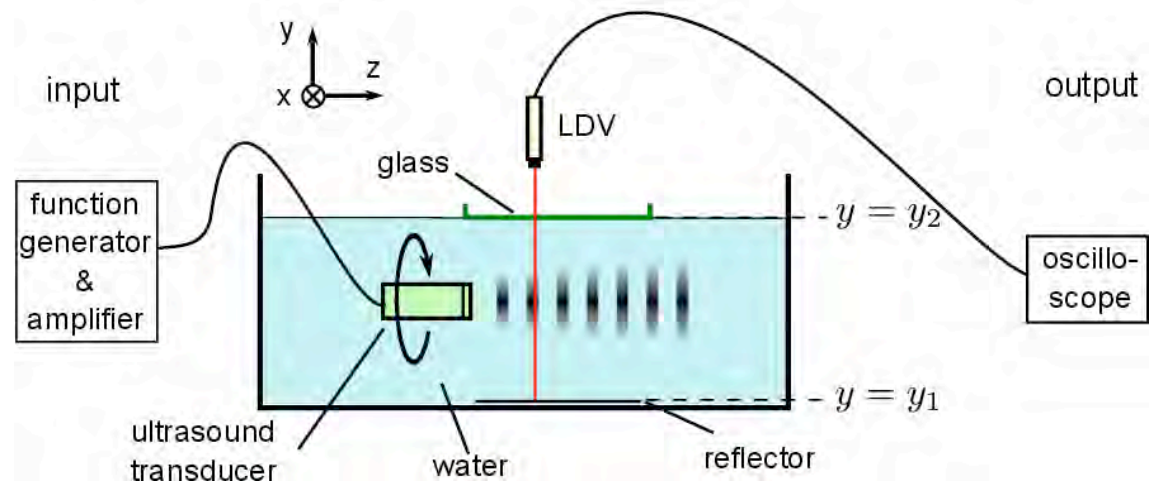

Fig. 1: Sketch of the measurement setup.

\subsection{Tomographic reconstruction}

Since the change of optical path length $\Delta L$ can be measured by the laser Doppler vibrometer, there is only one unknown variable left in (1). To solve such an integral equation, the well-developed filtered back projection (FBP) is chosen, which provides reliable results even in case of low signal-to-noise ratios (SNR) [1]. The FBP-algorithm can be shortly described as follows: For a two-dimensional reconstruction, the spatial distribution $f(x, y)$ needs to be projected under $K$ different angles. Each projection $p_{\theta i}$ consists of $M$ sampling points equidistantly located on the $\xi$-axis (Fig. 2), which passes though the origin at angle $\theta_{i}$. Then, the spatial distribution $f(x, y)$ can be reconstructed

with

$$
f(x, y)=\frac{\pi}{K} \sum_{i=1}^{K} Q_{\theta_{i}}\left(x \cos \theta_{i}+y \sin \theta_{i}\right)
$$

$$
\begin{aligned}
Q_{\theta_{i}}(m \Delta \xi)=\Delta \xi \cdot \mathrm{FFT}^{-1}\{\operatorname{FFT} & \left.\left\{p_{\theta_{i}}(m \Delta \xi) \cdot h(m \Delta \xi)\right\}\right\} \\
\text { for } m & =-\frac{M}{2}, \ldots,-1,0,1, \ldots, \frac{M}{2}-1 .
\end{aligned}
$$

Here, FFT \{\} and $\mathrm{FFT}^{-1}\{\}$ indicate one dimensional Fourier- and inverse Fourier-Transform, respectively. $\Delta \xi$ is the distance between two adjacent sampling points and $h()$ denotes a convolution kernel in the spatial domain, e.g., the Ram-Lak kernel in the following experiments [2].

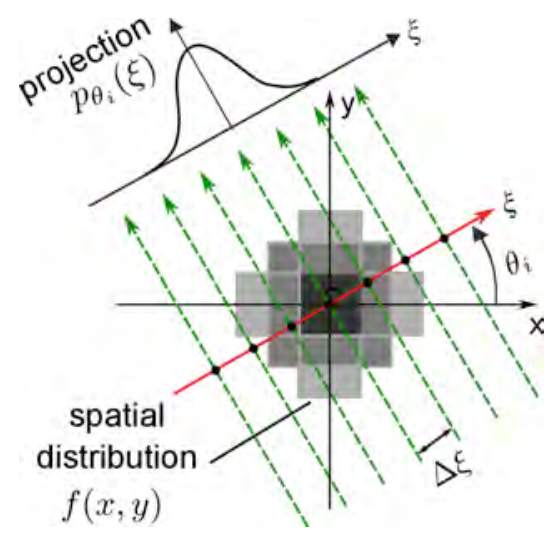

Fig. 2: Projection of spatial distribution $f(x, y)$ at angle $\theta_{i}$. 
In our case, the spatial distribution $f(x, y)$ denotes the change of refractive index $\Delta n(x, y)$ on a certain cross section $z=z_{0}$ and at instant of time $t=t_{0}$.

\subsection{Sound pressure in liquid}

Although (1) is valid in any transparent medium, the term, the change of refractive index, is quite unusual for describing ultrasound fields. According to the piezo-optic effect, the change of refractive index $\Delta n$ in gas or liquid is proportional to the ultrasound pressure

$$
\Delta n(x, y, z, t)=\left(\frac{\partial n}{\partial p}\right)_{S} p(x, y, z, t)
$$

where $(\partial n / \partial p)_{s}$ is the so-called piezo-optic coefficient (e.g., $1.444 \cdot 10^{-10} \mathrm{~Pa}^{-1}$ at $25^{\circ} \mathrm{C}$ in water) describing the variation of refractive index with pressure, and is assumed to be linear over the small range usually involved [3].

\subsection{Density change in transparent solid}

In contrast to the pressure field in liquids, the sound field in solid can only be completely expressed by vector functions (e.g., mechanical stress $\sigma$ ) [4], which are usually not able to be derived from the scale function (i.e., the change of refractive index $\Delta n$ ) without introducing significant errors. But according to the Lorentz-Lorenz-equation, we can convert the change of refractive index $\Delta n$ into the change of density $\Delta \rho$ using the following relationship

$$
R_{\mathrm{LL}}=\frac{n(x, y, z, t)^{2}-1}{n(x, y, z, t)^{2}+2} \cdot \frac{1}{\rho(x, y, z, t)}=\mathrm{const} \text {, }
$$

with

$$
\left\{\begin{array}{l}
n=n_{0}+\Delta n \\
\rho=\rho_{0}+\Delta \rho
\end{array}\right.
$$

Here, $n_{0}$ and $\rho_{0}$ denote the refractive index and the density of the medium in equilibrium state, respectively. Their values are usually available for common materials. The Lorentz-Lorenz-equation is derived from the Maxwell's equations under the assumption, that the electric polarizability of the atoms or molecules of the medium is constant [5]. Although this assumption can only be fulfilled in idealized materials, the deviations of the Lorentz-Lorenz specific refraction $R_{\mathrm{LL}}$ measured in experiments are rarely more than $1 \%$ even under extreme conditions of temperature and pressure [6]. As a result, we use the relative change of density (i.e., $\Delta \rho / \rho_{0}$ ) in solid to visualize the ultrasound field.

\section{Experimental Setup}

In the experiments, a cylindrically focused ultrasound transducer (Olympus V306) was immersed in water and excited by a $1 \mathrm{MHz}$ burst signal sent every $400 \mu \mathrm{s}$. For the measurement of optical path length change $\Delta L$, a differential LDV (Polytech OFV 512) was used, because it has much lighter sensor head comparing to that of single point laser Doppler vibrometer (e.g., Polytec OFV 303 used in our previous experiments [7]). During the measurement, the sensor head was mounted on a linear positioning unit (Physik Instrumente M-531.DG) and continuously transfered to different sampling positions. Consequently, the reduced weight induced much weaker and shorter mechanical vibrations after each transfer and led to a reduction of measurement duration for each scanning plane from 7 hours to 4 hours in our case. In order to project the ultrasound field from different angles, the ultrasound transducer was rotated by a precise rotation unit (Physik Instrumente M-037.DG) around its middle axis. Compared to the rotation of sensor head around the whole water basin, the rotation of transducer is mechanically much easier to implement. Besides, the media, where the ultrasound propagates, remain stationary during the whole measurement, thus, the optical path can be easily determined, even if several materials are involved (e.g., water, air and PMMA in our case). On the other hand, this implementation is obviously restricted to measuring ultrasound arising from a rotatable transducer. Due to the high sensitivity of laser beam to the flatness of water surface, a piece of glass was installed horizontally on the water surface to 
suppress any unwanted influences (e.g., vibration or fibers). The output signal of the LDV was acquired by an oscilloscope (Tektronix TDS 3054) with $100 \mathrm{MHz}$ temporal sampling rate, and subsequently transferred to a computer via GPIB bus. To enhance the SNR, single averaging of 32 measurements was carried out within the oscilloscope.

For the visualization of ultrasound field, the experimental setup keeps the same, except that, a PMMA cuboid of $50 \mathrm{~mm} \times 100 \mathrm{~mm} \times 200 \mathrm{~mm}$ was installed on the top of the optical reflector (Fig. 3). The surfaces of the PMMA cuboid have been polished. Because of the similar refractive indices of the laser light in water $(n=1.33)$ and PMMA $(n=1.49)$ at $20^{\circ} \mathrm{C}$, the laser beam can still be reflected back to the sensor head, even if the top and bottom surfaces of the cuboid are not exactly parallel to the water surface. On the contrary, the speed of sound in PMMA $(c=2760 \mathrm{~m} / \mathrm{s})$ is much faster than that in water $(c$ $=1484 \mathrm{~m} / \mathrm{s}$ ), that means, when the front surface of the PMMA cuboid is not perpendicular to the rotation axis of the transducer, the rotation axis of the ultrasound field in PMMA does not match that of the transducer any more. As a result, special care must be taken during the placement of the PMMA cuboid. Otherwise, significant error will be induced, because of the usage of inaccurate data about the rotation axis of ultrasound field.

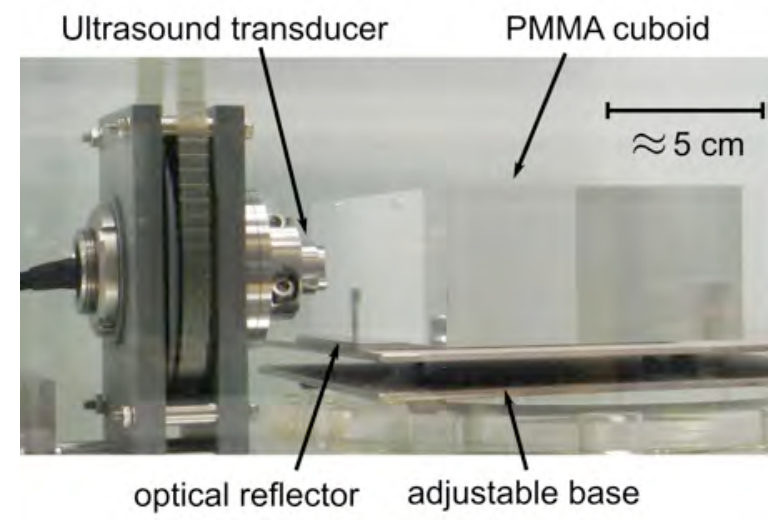

Fig. 3: Part of experimental setup for the measurement of density change within a PMMA cuboid induced by ultrasound.

To verify the reliability of this optical method, the same ultrasound pressure fields in water were also measured by means of a hydrophone (ONDA HNP-0400) with an electrode aperture of $0.4 \mathrm{~mm}$. These measurements were carried out under the conditions as close as possible as that using LDV.

\section{Experimental Results}

\subsection{Ultrasound pressure field in water}

The cylindrically focused ultrasound transducer under investigation has a radius of $6.35 \mathrm{~mm}$ and a focus length of $25.4 \mathrm{~mm}$. For the purpose of comparing two methods, the ultrasound pressure field underwater at $25.4 \mathrm{~mm}$ apart from the transducer front was measured. For the reconstruction, this cross section was sampled horizontally every $0.2 \mathrm{~mm}$ from $-20 \mathrm{~mm}$ to $20 \mathrm{~mm}$, and under 100 different angles, i.e., $\Delta \xi=0.2$ $\mathrm{mm}, M=200$ and $K=100$ in (2). The choice of the parameters were based on a FEM-simulation of ultrasound pressure field, which describes the theoretical distribution of the ultrasound pressure. The measurement took altogether about 4 hours, while the subsequent reconstruction of the pressure field at 2000 different instants of time required less than 2 minutes on a commercial computer using multi-core parallel processing. After that, an area of $24 \mathrm{~mm} \times 24 \mathrm{~mm}$ on the same cross section was sampled by means of the hydrophone. The measurement lasted about 8 hours, because of the strong and long mechanical vibration after each displacement of the hydrophone.

The pressure amplitude distributions obtained by the LDV as well as the hydrophone are depicted in Fig. 4. The images show good agreement with each other and share many details in common even on their peripheries. For the sake of simplification, the pressure distributions along $x$ - and $y$-axes are compared in 
Figs. 5 and 6, respectively. It can be seen, that the differences in both directions exhibit a maximum of about $10 \%$.

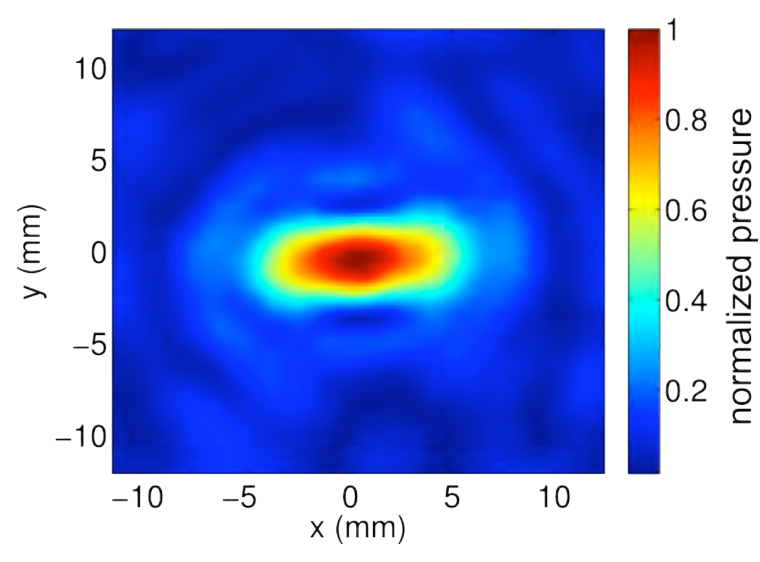

(a)

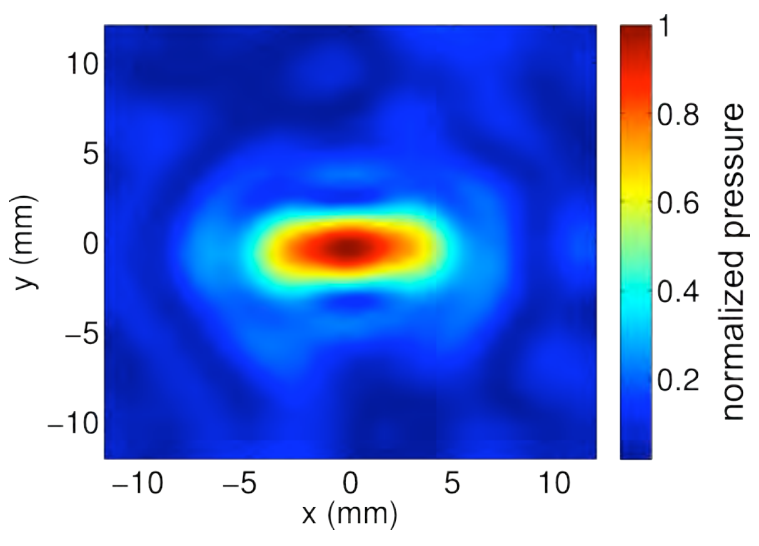

(b)

Fig. 4: Pressure amplitude distribution at $25.4 \mathrm{~mm}$ apart from the transducer front.

(a) reconstructed by means of optical method. (b) measured using hydrophone.
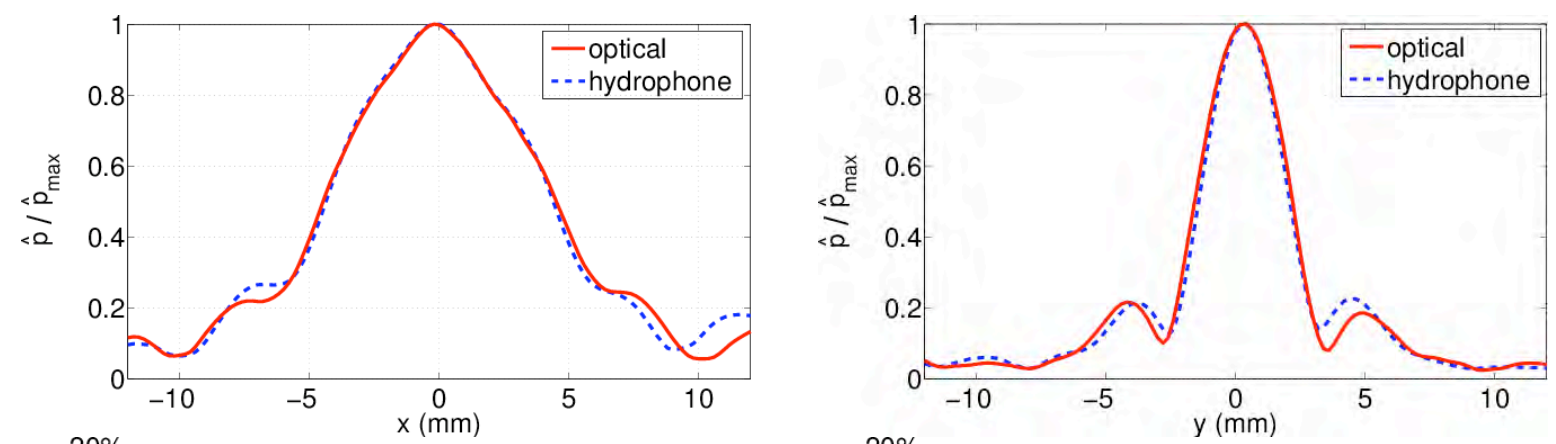

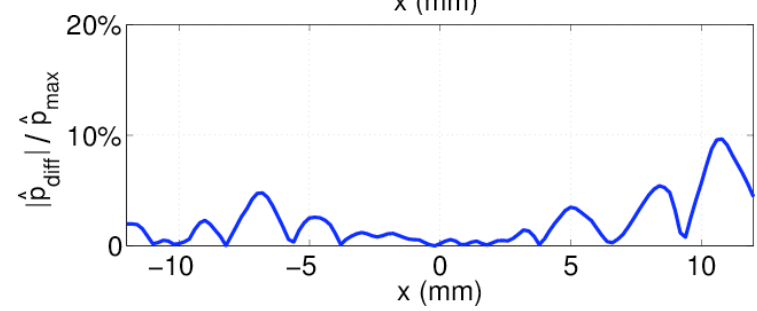

Fig. 5: Comparison of the pressure amplitude distribution along $x$-axis (i.e., $y=0$ ) in Figs. (4a) and (4b).

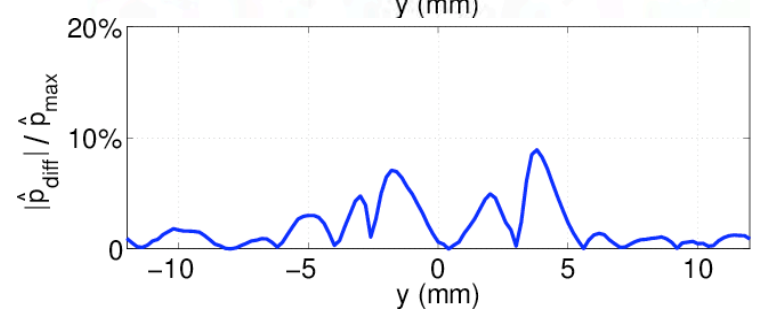

Fig. 6: Comparison of the pressure amplitude distribution along $y$-axis (i.e., $x=0$ ) in Figs. (4a) and (4b).

\subsection{Change of density in PMMA}

In this experiment, the PMMA cuboid was installed at $10 \mathrm{~mm}$ apart from the transducer front. We utilized the laser beam from the LDV to make sure, that the rotation axis of the transducer was perpendicular to the front surface of PMMA cuboid. As an example, the change of density at $20 \mathrm{~mm}$ apart from the transducer front was reconstructed. For the measurement of optical path length change, the same parameters were used as in the last one. After the reconstruction of refractive index change on the whole cross section, the relative density change $\Delta \rho / \rho_{0}$ can be directly calculated using (4) (Fig. 7). 
To the best of our knowledge, there is still no other method published, which can quantitatively reconstruct the change of density induced by ultrasound within a PMMA cuboid. Consequently, we are not able to verify the reliability of the results. But if we compare the distribution of the ultrasound pressure field in water (Fig. 4) and the distribution of the density change in PMMA (Fig. 7) arising from the same source, the images in the center are pretty similar.

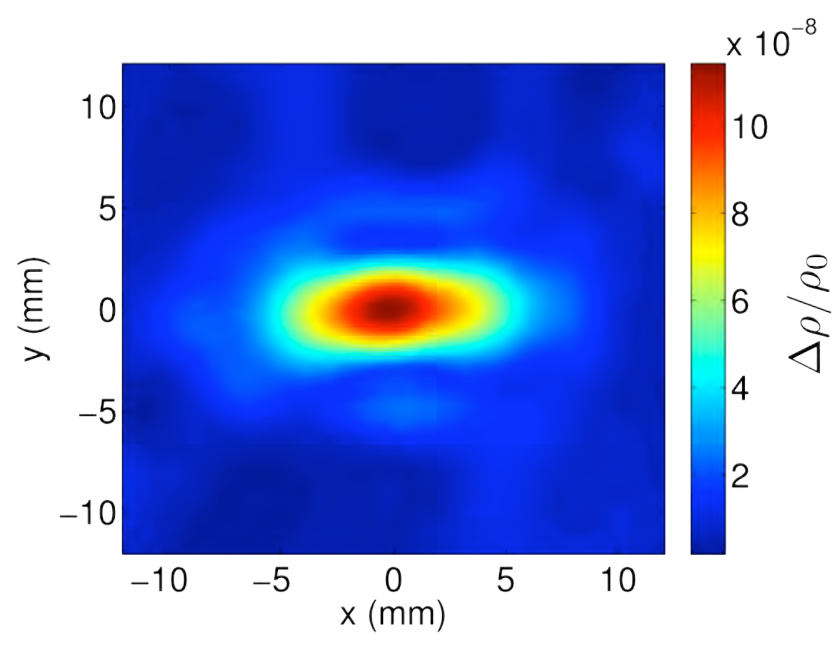

Fig. 7: Relative change of density in PMMA on the cross section at $20 \mathrm{~mm}$ apart from the transducer front caused by the ultrasound.

\section{Conclusion}

In this paper, we utilized a differential LDV to measure the change of optical path length induced by the ultrasound field in water and PMMA. After that, we successfully reconstructed the ultrasound pressure field in water and density change in PMMA. The former one shows good agreement with that obtained by hydrophone, while the later one is a novel research and its results seem already quite plausible.

After confirming the reliability of this method underwater, we can use its advantages, e.g., invasive measurement and omnidirectional characteristic, in some situations, where the hydrophone may fail. For example, the measurement of reflected ultrasound directly before a boundary. Besides, since we have now the ability to obtain the density change caused by ultrasound everywhere inside transparent media, lots of novel studies can be done in the future, e.g., the visualization of the propagation of ultrasound in solid.

\section{Acknowledgement}

The authors gratefully acknowledge funding of the Erlangen Graduate School in Advanced Optical Technologies (SAOT) by the German Research Foundation (DFG) in the framework of the German excellence initiative.

\section{REFERENCES}

[1] L. Bahr and R. Lerch, "Beam profile measurements using light refractive tomography," IEEE Trans. Ultrason., Ferroelectr., Freq. Control, vol. 55, no. 2, pp. 405-414, 2008.

[2] A. Kak and M. Slaney, Principles of Computerized Tomographic Imaging. Society of Industrial and Applied Mathematics, 2001.

[3] C. B. Scruby and L. E. Drain, Laser Ultrasonics. Adam Hilger, 1990.

[4] R. Lerch, G. Sessler, and D. Wolf, Technische Akustik. Springer Berlin Heidelberg, 2009. 
[5] M. Born, E. Wolf, A. Bhatia, P. Clemmow, D. Gabor, A. Stokes, A. Taylor, P. Wayman, and W. Wilcock, Principles of Optics: Electromagnetic Theory of Propagation, Interference and Diffraction of Light. Cambridge University Press, 2000.

[6] V. O. Bodmann, " Partielle spezifische Refraktionen von Polymethylmethacrylat und Polystyrol. I. Einfluss verschiedener loesungsmittel," Die Makromolekulare Chemie, vol. 122, no. 1, pp. 196-209, 1969.

[7] L. Chen, S. J. Rupitsch, P. Winter, and R. Lerch, "Reconstruction of sound pressure fields of ultrasound transducers by means of computed tomography," in Proceedings of the 20th IMEKO TC2 Symposium on Photonics in Measurement, 2011. 